\title{
Tractable lithium-ion storage models for optimizing energy systems
}

\author{
Fiodar Kazhamiaka* (D), Catherine Rosenberg and Srinivasan Keshav
}

\author{
${ }^{*}$ Correspondence: \\ fkazhami@uwaterloo.ca \\ University of Waterloo, 200 \\ University Ave. W, N2L 3 G1 \\ Waterloo, ON, Canada
}

\begin{abstract}
Model-based optimization of energy systems with batteries requires a battery model that is accurate, tractable, and easy to calibrate. Developing such a model is challenging because electrochemical batteries exhibit complex behaviours. In this paper, we propose and evaluate a family of battery models that have different trade-offs between accuracy and complexity. We derive our models from a recently developed battery model, which is accurate and easy to calibrate but is not tractable. We evaluate our models against the commonly-used benchmark tractable model using a set of experiments that characterize the cycling behaviour of two Lithium-ion battery chemistries, as well as dynamic charge/discharge experiments. We further compare the models for two typical energy system applications, solar farm firming and grid regulation, to show the impact of the choice of battery model on the results. Finally, we evaluate the increase in accuracy when battery models are calibrated with the proper operating range.
\end{abstract}

Keywords: Battery model, Optimization, Energy

\section{Background}

The rapid decline in the cost of energy storage, particularly Lithium-ion batteries, has motivated a wide range of storage applications (Scrosati et al. 2011). Many of these applications, such as grid support and home energy management, require large, expensive battery systems. Hence there are many research studies being conducted on optimal battery dimensioning and operation using model-based analysis of the energy system under consideration.

There exists several models which can accurately take into account the dynamic behaviour of Lithium-ion cells, including their non-linear properties such as voltage hysteresis, efficiency losses, degradation, and temperature effects. A cell model has to be combined with a battery management system (BMS) model, which captures voltage, current, and temperature limits, to get a complete dynamic battery system model. Most of the cell models use an equivalent circuit to emulate model behaviour (see He et al. (2011); Hu et al. (2012); Chen and Rincon-Mora (2006) for examples) or complex equations to represent the electrochemical interactions inside the battery (see Klein et al. (2013); Tanim et al. (2015) for examples). Some of these models are difficult to calibrate because they require data which is onerous to obtain (i.e., can only be obtained via experiments). For example, RC circuit models (Chen and Rincon-Mora 2006; Hu et al. 2012) require pulse charge/discharge measurements to set the resistance and capacitance parameters of the

(c) The Author(s). 2019 Open Access This article is distributed under the terms of the Creative Commons Attribution 4.0 International License (http://creativecommons.org/licenses/by/4.0/), which permits unrestricted use, distribution, and reproduction in any medium, provided you give appropriate credit to the original author(s) and the source, provide a link to the Creative Commons license, and indicate if changes were made. 
circuit, and while there are attempts to reduce the calibration effort of these models (Einhorn et al. 2013; Hentunen et al. 2014; Jackey et al. 2013), calibrating them is still not an easy task. Furthermore, these models are described using complex mathematical functions such as differential equations, and this makes them unsuitable for optimization studies using mathematical programs.

The computational effort required to solve a mathematical program is proportional to the number and complexity of equations and model constraints in the program. The simplest programs to solve are linear programs (LP), which have reasonable solving times even when the program has hundreds of thousands of constraints (Gearhart et al. 2013). The most commonly used battery model in studies requiring an optimization framework is linear (for examples, see Khalilpour and Vassallo (2016); Hassan et al. (2017); Mehrabankhomartash et al. (2017); Atia and Yamada (2016); Chen et al. (2012)). It uses power as input, rather than current. This model, henceforth referred to as the benchmark, uses linear and constant approximations to many of the non-linear dynamic characteristics of a Lithium-ion battery, and the loss in model accuracy as a result of these approximations is not clear. We are unaware of any published works that thoroughly evaluate the benchmark model's accuracy, explain how to calibrate it properly, and explore how it affects the results of the optimization studies that use it.

In this paper, we start by comparing the accuracy of the benchmark to an experimentally-validated, non-linear, and non-analytic PI model ${ }^{1}$ developed by Kazhamiaka et al. (2018) which is also power-based (power is conserved, and powerbased models are much simpler since they do not necessarily require voltage/current transformations). To do so, we propose a calibration process for the benchmark and we find that it compares poorly with the PI model in estimating the battery energy content, which suggests that it is problematic to base battery optimization studies on this model. This finding motivates the development of new battery models with improved accuracy to replace the benchmark as the go-to model for mathematical programming. Developing and validating these new models is the main focus of this paper. In this context, we aim at developing models with the following desirable properties:

1. Tractability: use only simple polynomial expressions with low degrees. The goal is to be as simple as possible to reduce the computation time, while respecting the inherent non-linearity of Lithium-ion batteries.

2. Calibration using only the data commonly found in a battery datasheet (spec), to avoid onerous experiment-based calibration and therefore make it more likely for the model to be adopted by the research community.

3. Use power as input, rather than current.

4. Integrate the constraints typically imposed by a BMS, i.e., a complete battery model, rather than a cell model.

Similar to the work done by Zou et al. (2016) to extract simpler models from a highorder physics-based Lithium-ion battery model and reduce the computational effort of simulations, in this paper we start with the non-analytical PI model, which has the last three of the above properties, and extract models that have all the four desirable properties. The methodology developed here can easily be reused for other battery chemistries. Our contributions are as follows: 
- We evaluate the accuracy of the benchmark model and incidentally show how to calibrate it.

- We methodically extract three tractable models, one of which is linear, from the PI model and explain how to calibrate them using a spec sheet.

- We thoroughly evaluate each of them using an extensive measurement campaign on two Lithium-ion battery chemistries.

- We conduct two application studies that illustrate the effect of model complexity on the outcome of optimization analyses.

- We provide guidelines for researchers on how to choose which model to use in order to improve the accuracy of their battery system study.

The main messages of our study are the following:

- We have developed a new linear model that is more accurate and just as tractable as the benchmark model for optimization studies.

- The choice of model can significantly affect the conclusions of model-based energy system studies and that sometimes a quadratic model is necessary.

- The operating range of the battery is a crucial parameter for obtaining accurate results with simplified models.

The rest of the paper is organized as follows. In the "Existing models" section we describe the benchmark and PI models. In "A Family of Tractable Models" section, we simplify the complex aspects of the PI model to develop and calibrate a set of tractable models. In the "Evaluation" section, we compare the accuracy of the tractable models, the benchmark and the PI model. In the "Applications" section, we dimension a solar farm and a grid regulation battery application using different models to characterize the practical trade-off between the accuracy and complexity of our tractable models. We provide some practical advice on how to choose the right model for a system study In the "Discussion and Limitations" section and conclude the paper in the "Conclusion" section.

\section{Existing models}

In the following, we use a discrete time model. The duration of a time slot is $T_{u}$ and the time index is $k$. Table 1 summarizes the notations and the corresponding units.

Table 1 Notation

\begin{tabular}{lll}
\hline Name & Description & Unit \\
\hline$P(k)$ & Power applied to battery in time slot $k$ & W \\
$I(k)$ & Current estimate for time slot $k$ & $\mathrm{~A}$ \\
$V(k)$ & Battery voltage estimate for time slot $k$ & $\mathrm{~V}$ \\
$b(k)$ & Battery energy content estimate at the end of time slot $k$ & Wh \\
$a_{1}\left(a_{2}\right)$ & Minimum (maximum) cell energy content & Wh \\
$\alpha_{C}\left(\alpha_{d}\right)$ & Charge (discharge) current limits per unit of storage & A/Wh \\
$\eta_{c}\left(\eta_{d}\right)$ & Charge (discharge) inefficiency & $\mathrm{V}$ \\
$M$ & Voltage function that uses energy content and current as input & $\Omega$ \\
$n$ & Number of cells that compose the battery & $\mathrm{h}$ \\
$R_{i c}\left(R_{i d}\right)$ & Internal cell impedance during charging (discharging) & \\
$T_{u}$ & Time slot duration & \\
\hline
\end{tabular}




\section{The Benchmark Model}

Given the energy content, $b(k-1)$, of a battery in time-slot $k-1$, and an applied power $P(k)$ which is constant over time slot $k$, the benchmark model calculates an estimate of the energy content $b(k)$ at the end of the $k^{\text {th }}$ time slot using simple linear equations. It models three basic properties of a cell: 1$)$ the charging and discharging inefficiencies $\left(\eta_{d}\right.$ and $\eta_{c}$, respectively), 2) the upper and lower energy content limits ( $a_{1}$ and $a_{2}$, respectively), and 3) the charging and discharging current limits $\left(\alpha_{c}\right.$ and $\alpha_{d}$, respectively), which are converted into power limits by taking their product with the corresponding nominal cell voltage ( $V_{n o m, c}$ and $\left.V_{n o m, d}\right)$. The (constant) values of all these parameters can be obtained from the spec sheet of the cell. For a battery system comprised of $n$ cells, the battery energy and power limits scale with $n$.

The typical formulation of the benchmark model for a battery with $n$ cells is given below. The piece-wise Eq. 2 is often expressed in linear form by splitting $P(k)$ into separate charging and discharging variables, say $c(k)$ and $d(k)$ respectively. It is physically impossible for the model to charge and discharge the battery simultaneously, which yields the following non linear constraint $c(k) \cdot d(k)=0$. It has been shown in Ghiassi-Farrokhfal et al. (2016) that this constraint can often be relaxed, making the model linear.

\section{The benchmark ( $n$ cells):}

$$
\begin{aligned}
& b(k)=b(k-1)+\Delta_{E}(k) \\
& \Delta_{E}(k)=\left\{\begin{array}{l}
\eta_{c} P(k) T_{u}: P(k) \geq 0 \\
\eta_{d} P(k) T_{u}: P(k)<0
\end{array}\right. \\
& n \alpha_{d} V_{n o m, d} \leq P(k) \leq n \alpha_{c} V_{n o m, c} \\
& n a_{1} \leq b(k) \leq n a_{2}
\end{aligned}
$$

Note that $\eta_{c}, \eta_{d}, a_{1}$, and $a_{2}$ are all constant, and the performance of the model is heavily dependent on the calibration of these parameters. However, it is common practice to calibrate the model without considering the following: 1) the operating range of the application (discussed in the "Operating Range" section), and 2) all of the data typically available in a spec sheet. In most papers, the following parameters are used for lithium-ion batteries irrespective of the operating range: the discharging efficiency is equal to the charging efficiency and is in the range of 90-95\%, and $a_{1}=0, a_{2}=B$ where $B$ is a 'nominal' capacity. We will show that the benchmark model can be derived from the PI model, and hence the calibration procedure we propose is based on the calibration of the PI model, where parameter values are computed from spec sheet data. We discuss parameter calibration in greater detail in the "Methodology" and "Operating Range" sections.

A significant shortcoming of this model is that, in reality, both the efficiencies and energy content limits of a battery are dependent on the current being applied, and the efficiencies also depend on the battery voltage. The benchmark model does not have an internal representation of the voltage and current, which hinders its accuracy; we evaluate model accuracy in the "Evaluation" and "Applications" section. The more accurate PI model, which models these dependencies, is discussed next. 


\section{The PI Model}

The purpose of a dynamic battery model is to compute the evolution of the energy content of a battery as it is charged or discharged. However, it is extremely difficult to directly measure the energy content of a battery. The PI model takes advantage of the relationship between battery voltage and current, which are easy to measure, to infer the energy content. The full formulation, calibration procedure, and evaluation of the PI model can be found in Kazhamiaka et al. (2018). All of our tractable models are derived from the PI model, so we describe it in detail below.

The inputs to the PI model are the applied power $P(k)$, and the energy content of the battery in the previous time step, $b(k-1)$. The model calculates an estimate of the resulting battery $V(k)$, charge/discharge current $I(k)$, and energy content $b(k)$. It takes into account the voltage- and current-dependent charging and discharging inefficiencies $\left(\eta_{c}(I(k))<1\right.$ and $\eta_{d}(I(k))>1$, respectively), the current-dependent lower and upper limits on the energy content $\left(a_{1}(I(k))\right.$ and $a_{2}(I(k))$, respectively), as well as the charging and discharging current limits ( $\alpha_{c}$ and $\alpha_{d}$, respectively).

The voltage of the battery is modeled using a function $M$ which maps the energy content and applied current to a unique battery voltage. The $M$ function captures the voltage hysteresis behaviours, where the voltage jumps relative to the open-circuit voltage (idle battery) when a charging current is applied, and drops when a discharging current is applied. The $M$ function also captures the proportional change in voltage with respect to the change in the battery's energy content.

The functions $a_{1}(),. a_{2}($.$) , and M($.$) and constants \alpha_{c}$ and $\alpha_{d}$ are parameters to the model and can be obtained using a spec sheet. $\eta_{c}($.$) and \eta_{c}($.$) are given by Eqs. 7$ and 8 respectively. They are functions of $I$ and $V$ as well as the internal impedances of a cell $R_{i c}$ and $R_{i d}$ for charging and discharging respectively, which can also be found in a spec sheet ${ }^{2}$.

The following set of equations and constraints describes the PI model:

\section{The PI model:}

$$
\begin{aligned}
& b(k)=b(k-1)+\Delta_{E}(k) \\
& \Delta_{E}(k)=\left\{\begin{array}{l}
\eta_{c}(I(k), V(k)) P(k) T_{u}: P(k) \geq 0 \\
\eta_{d}(I(k), V(k)) P(k) T_{u}: P(k)<0
\end{array}\right. \\
& \eta_{c}(I(k), V(k))=1-\frac{I(k) R_{i c}}{n V(k)}: I(k) \geq 0 \\
& \eta_{d}(I(k), V(k))=1-\frac{I(k) R_{i d}}{n V(k)}: I(k)<0 \\
& V(k)=M(b(k), I(k)) \\
& I(k)=\frac{P(k)}{V(k)} \\
& n \alpha_{d} \leq I(k) \leq n \alpha_{c} \\
& a_{1}(I(k)) \leq b(k) \leq a_{2}(I(k))
\end{aligned}
$$


This formulation requires the numerical computation of the intersection between the surface defined by Eq. 9 in the plane $(I(k), V(k))$ and the surface (in the same plane) defined by the following equation (assuming $P(k) \geq 0$ ):

$$
b(k)=b(k-1)+\left(1-\frac{I(k) R_{i c}}{n V(k)}\right) I(k) V(k) T_{u}
$$

The details of this process, as well as instructions on how to calibrate the model parameters, are documented in Kazhamiaka et al. 2018.

The $a_{1}(),. a_{2}(),. M(),. \eta_{c}($.$) , and \eta_{d}$ (.) functions are shown for a Lithium-Titanate (LTO) cell (Leclanché 2014) in Fig. 1, where the current is expressed in terms of C-rate; $1 \mathrm{C}$ is defined as the current which fully discharges the battery in one hour. The cell has a nominal charge capacity of $30 \mathrm{Ah}$, hence $1 \mathrm{C}$ corresponds to a $30 \mathrm{~A}$ current. A negative C-rate is used to represent a discharging current.

\section{A Family of Tractable Models}

The PI model has three principal components: the $M$ function that maps from energy content and charge/discharge current to battery voltage, the energy content limits $\left(a_{1}()\right.$. and $\left.a_{2}().\right)$, and the inefficiency functions $\left(\eta_{c}(\right.$.$\left.) and \eta_{d}().\right)$. The $M, a_{1}$, and $a_{2}$ functions are numerically derived from the spec sheet, i.e., they are not explicit, and this prevents the PI model from being used as part of a mathematical optimization program. The inefficiency functions are explicit but yield non-linearities and this makes the model difficult to use for mathematical optimization.

We therefore derive a set of tractable models by approximating these three components with polynomials. Our methodology is to test different combinations of approximations, starting with higher degree polynomials and working down to constant approximations, keeping combinations that show a noticeable difference in performance compared to the next iteration, and discarding those that do not have a favourable accuracy-complexity trade-off. We test each combination by simulating charge/discharge cycles at different $C$-rates and comparing the $b(k)$ estimates of the model with that of the PI model to quantify the loss of accuracy due to the approximations. The complexity of each model is on the spectrum between the PI and benchmark models; indeed, approximating the three complex components of the PI model with constants results in the benchmark model. In developing our models, we experimented with polynomial approximations of degree less than or equal to 3 .

In this section, we show how to derive the benchmark model and three novel tractable models which have a favourable accuracy-complexity trade-off.

\section{Methodology}

We now discuss our analytical approximations to voltage, efficiency, and energy content limit functions; these are functions of the current, which is estimated from the input power using Eq. 10. We compute the parameters to each approximation to minimize the error between the PI model's version of the corresponding function and the approximation for a given operating range (OR), which corresponds to the maximum range of permissible currents as defined by the spec sheet or programmed into the BMS by the system operator (we elaborate on the OR in the "Operating Range" section). Our intuition is that this approach minimizes the error introduced by the approximation. Each approximation 


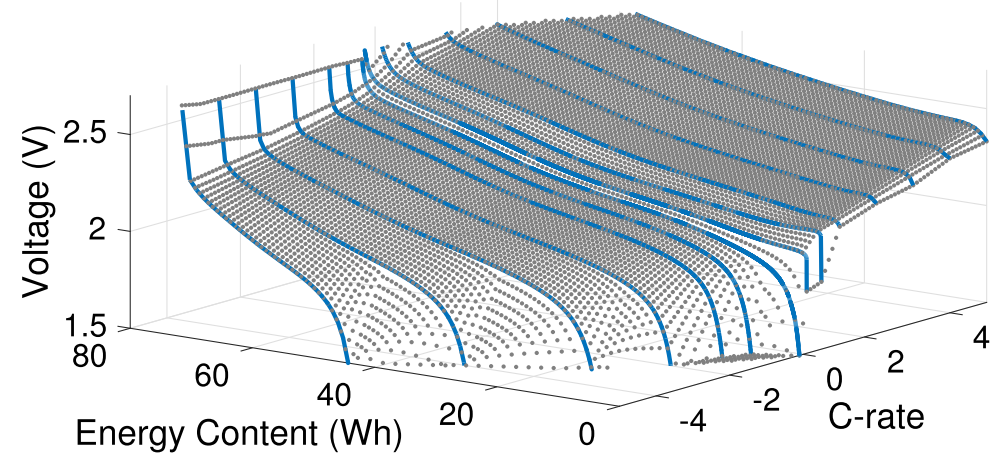

(a) $M(b(),. I()$.$) function$

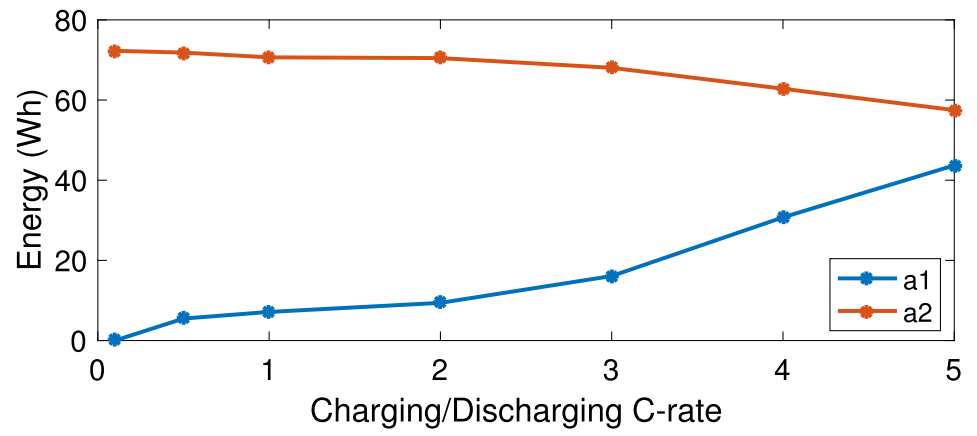

(b) $a_{1}(),. a_{2}($.$) energy content limits$
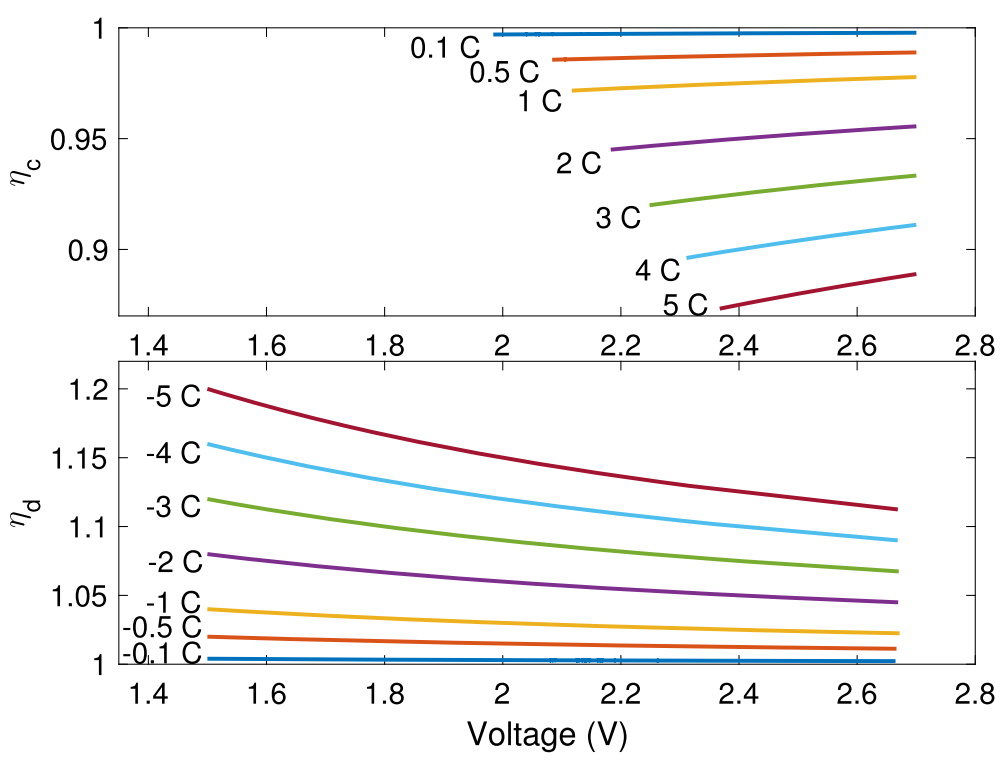

(c) Efficiencies $\eta_{c}(I(),. V()$.$) and \eta_{d}(I(),. V()$.

Fig. 1 a $M$ function, which maps the applied current and energy content to a unique voltage, shown with blue curves obtained from a spec and grey points representing the interpolated surface between the curves. b $a_{1}($.$) and a_{2}($.$) : points obtained from the spec. \mathbf{c} \eta_{c}($.$) and \eta_{d}($.$) , with each line representing the efficiency$ at a different $\mathrm{C}$-rate, as per Eqs. 7 and 8

is evaluated by comparing the energy content estimates of the resulting model to the PI model over constant-current charge/discharge cycles and a varying charge/discharge profile. We give a detailed account of the evaluation in the "Evaluation" section. 


\section{Voltage Estimate}

When a battery has a certain amount of energy that can be extracted from it, and is subject to a current, this results in a certain voltage across the positive and negative electrodes. In the PI model, the voltage estimate is obtained by using the $M$ function, which represents the relationship between energy, current, and voltage for a given battery, and is obtained by sampling the voltage curves found in the battery's spec sheet. To make the PI model analytical, $M$ has to be expressed in analytic form. We consider the following two polynomial approximations ${ }^{3}$ :

$$
\begin{aligned}
& V(k)=\left\{\begin{array}{l}
V_{\text {nom }, d}: P(k)<0 \\
V_{\text {nom }, c}: P(k) \geq 0
\end{array}\right. \\
& V(k)=x_{00}+x_{10} I(k)+x_{01} b(k)
\end{aligned}
$$

The constant approximation of the voltage corresponds to the 'nominal' voltage of the battery, $V_{\text {nom,c }}$ when charging and $V_{\text {nom,d }}$ when discharging; these can be computed by taking the average of the voltage curves over the OR. Note that we can separate the charging voltage from the discharging voltage (as done in Eq. 13) for the linear approximation (Eq. 14) without increasing the complexity of the model because of the natural separation between charging and discharging processes in Eqs. 6, 11, and 12, but we found that this has little effect on the overall accuracy of the resulting model when applied to Eq. 14 .

Figure 2 shows the non-analytic $M$ function from the PI model, and the linear approximation given by Eq. 14 which takes the form of a plane with the best least-squares approximation of the function ${ }^{4}$ over the OR. The plane is able to capture the positive slope of the voltage with increasing current and energy content, but does not capture the

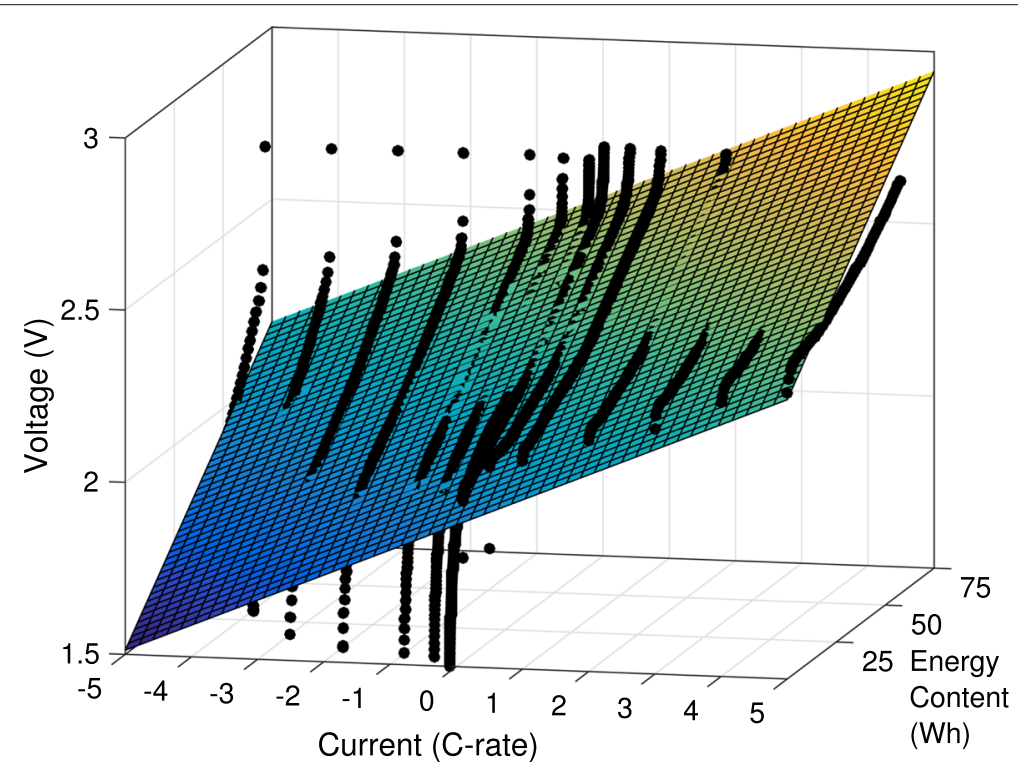

Fig. $2 M$ function, represented by the black dots which map the applied current and energy content to a unique voltage and are obtained from a spec sheet. The plane is a linear approximation to the $M$ function (Eq. 14) 
rapid drop as the battery voltage approaches the lower limit. A cubic function is able to capture these dynamics, however we found that the additional complexity of the cubic function over the plane offered negligible improvements to the accuracy of the model.

\section{Energy content limits}

Energy content limits are functions of the current, and can be obtained empirically from the voltage curves in the spec sheet. We consider the following approximations to $a_{1}($.) (similarly for $a_{2}($.$) ):$

$$
\begin{aligned}
& a_{1}(I(k))=n \overline{a_{1}} \\
& a_{1}(I(k))=u_{1} I(k)+v_{1} n
\end{aligned}
$$

$\overline{a_{1}}$ is computed by taking the average of the $a_{1}($.$) curve over the OR, and u_{1}$ and $v_{1}$ are parameters which define the line of best fit (least-squares) to $a_{1}($.) over the OR. In our evaluation, we found that the linear form in Eq. 16 offers the best trade-off between accuracy and complexity, i.e., better approximations offered very small improvement in accuracy at the cost of much higher complexity. Figure 3 shows the $a_{1}($.$) and a_{2}($.) functions for an LTO cell, as well as their linear least-squares and constant approximations used in our tractable models.

\section{Charging/discharging efficiencies}

In the PI model, the charging (resp. discharging) efficiency (Eqs. 7, 8) is a function of the voltage, current, and nominal internal resistance during charging $\left(R_{i c}\right)$ (resp. discharging $\left.\left(R_{i d}\right)\right)$. The efficiency formula makes the model inherently non-linear, since both $I$ and $V$ are variables of the model and hence the equation is quadratic in terms of the variables. Furthermore, the energy content update equation (Eq. 6) has a product of the efficiency and the applied power-which is the free variable in an optimization problem-to update

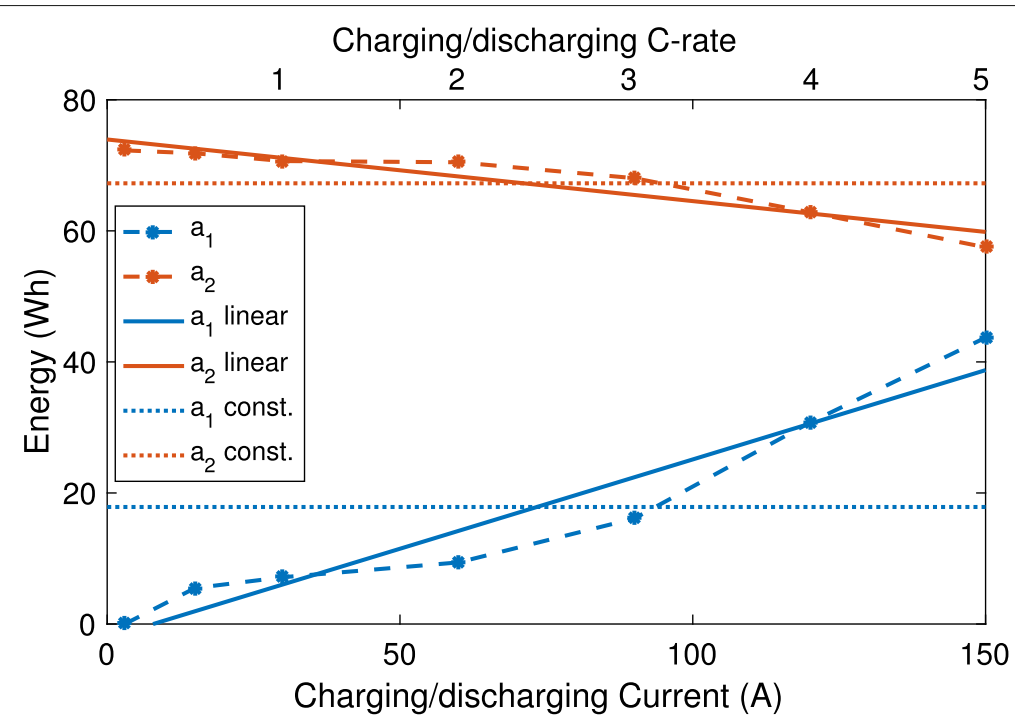

Fig. 3 Lower $\left(a_{1}\right)$ and upper $\left(a_{2}\right)$ energy limits for an LTO cell, along with linear and constant approximations computed over the maximum OR of the battery (up to $5 \mathrm{C}$ charging and discharging) 
the battery energy content estimate. We consider the following approximations for $\eta_{c}$ (similarly for $\left.\eta_{d}\right)$ :

$$
\begin{aligned}
& \eta_{c}=\bar{\eta}_{c} \\
& \eta_{c}(I(k))=1-\frac{I(k) R_{i}}{V_{\text {nom, },}}
\end{aligned}
$$

Using constant approximations for the charging $\left(\bar{\eta}_{c}\right)$ and discharging $\left(\bar{\eta}_{d}\right)$ efficiency linearizes this part of the model; they are calculated as the average values of $\eta_{c}(I, V)$ and $\eta_{d}(I, V)$ over the OR. Another approximation (Eq. 18) can be obtained by using $V_{\text {nom,c }}$ in place of $V(k)$ to reduce the dimensionality of the calculation, thereby reducing the non-linearities in the model.

To illustrate the efficiency functions and the effect of the approximations, the charging and discharging efficiencies of an LTO cell is shown in Fig. 4, with solid lines representing Eqs. 7 and 8, asterisks representing the efficiencies when calculated using a constant approximation of the charging voltage as $V_{n o m, c}$ and $V_{n o m, d}$, and squares representing constant efficiency approximations.
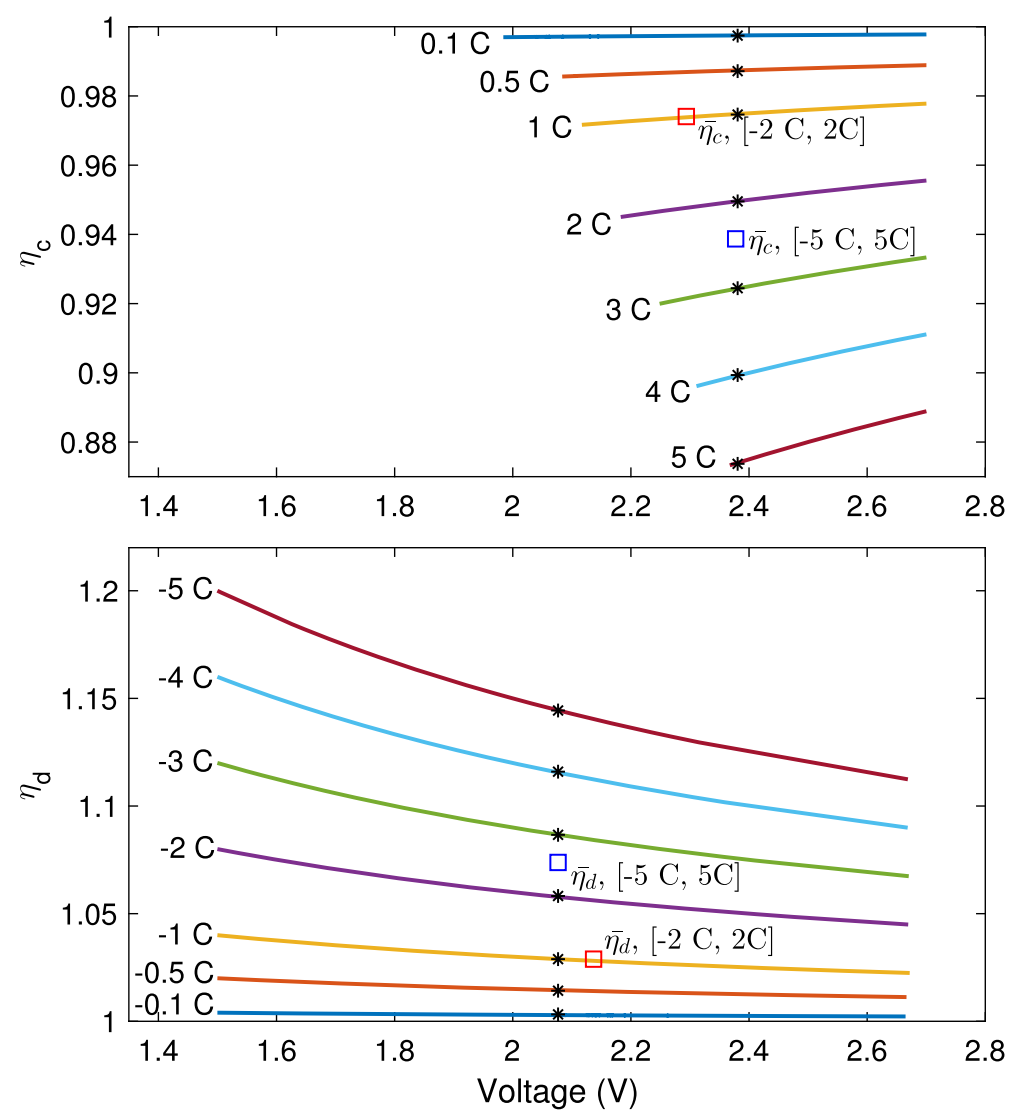

Fig. 4 The charging and discharging efficiencies of an LTO cell with $R_{i c}=R_{i d}=0.002 \mathrm{~m} \Omega$. Each solid line represents the efficiency calculated using Eqs. 7 or 8 at the labeled charging/discharging C-rate. Each asterisk represents the approximation where $V=V_{\text {nom, }}$ for charging and $V_{\text {nom, } d}$ for discharging. The constant approximations $\bar{\eta}_{c}$ and $\bar{\eta}_{d}$ are shown for two labeled operating ranges 


\section{Operating Range}

The operating range (OR) is an important parameter to consider when calculating the approximations to different curves. We define the OR as the range of currents that we expect to be applied to the battery. The intuition is that we should match the parameters of the modelled battery to reflect the actual operation of the battery, rather than calibrating the model to be as accurate as possible over the full OR capabilities of the battery. Indeed, some applications do not use the full OR. When the OR is narrow, we can expect a lower modelling error.

In Figs. 2, 3, and 4, the approximations to the corresponding functions were computed over the full operating range ([-5 C, $5 \mathrm{C}]$ for the LTO cell) unless otherwise noted. In this paper, we compute the constant approximations to be equal to the average value of their corresponding curve over the OR, and choose linear approximations that minimize the least-squared error to the curve over the OR. For example, Fig. 4 shows the constant approximation for charging efficiency $\left(\bar{\eta}_{c}\right)$ and discharging efficiency $\left(\bar{\eta}_{d}\right)$ for the full OR of $[-5 \mathrm{C}, 5 \mathrm{C}]$ as well as for a narrower OR of $[-2 \mathrm{C}, 2 \mathrm{C}] ; \bar{\eta}_{c}$ is higher for the narrower OR because the efficiency is higher at lower C-rates.

\section{Models}

We have developed notation to refer to each of the four models that we describe next, based on how the voltage, energy limits, and efficiencies are approximated. Similar to Kendall's notation for queues (Kendall 1953), each component of the model is described by a letter with slashes in between, i.e., Voltage function/Energy limit/Efficiency $(\mathrm{V} / \mathrm{E} / \eta)$ notation. A letter ' $Q$ ' means that that calculation uses a quadratic approximation, such as the voltage function in the PI model; ' $\mathrm{L}$ ' represents a linear approximation, and ' $\mathrm{C}$ ' a constant approximation ${ }^{5}$.

The four analytic models are summarized in Table 2, and we describe them below. Out of the four models, three approximate the voltage using two constants $V_{\text {nom,c }}$ and $V_{\text {nom,d }}$. For these three models, we can write their formulation in terms of power by replacing $I(k)>0$ by $P(k) / V_{n o m, c}$ and $I(k)<0$ by $P(k) / V_{n o m, d}$ in the PI model and can remove Eqs. 9 and 10 completely.

\section{$C / C / C$}

This model is exactly the benchmark summarized in Eqs. 1-4, and we henceforth refer to it as the $\mathrm{C} / \mathrm{C} / \mathrm{C}$ model. It uses constant approximations for efficiencies and energy

Table 2 Summary of Analytical Models

\begin{tabular}{|c|c|c|c|}
\hline \multirow[t]{2}{*}{ Model } & \multicolumn{3}{|l|}{ Approximations } \\
\hline & Voltage & Energy Content Limits & Efficiency \\
\hline \multirow{2}{*}{$\mathrm{C} / \mathrm{C} / \mathrm{C}$} & $V_{\text {nom, } \boldsymbol{d}}: P<0$ & $\bar{a}_{1} n$ & $\bar{\eta}_{d}$ \\
\hline & $V_{\text {nom }, \boldsymbol{c}}: P \geq 0$ & $\overline{\boldsymbol{a}}_{\mathbf{2}} n$ & $\bar{\eta}_{c}$ \\
\hline \multirow{2}{*}{$\mathrm{C} / \mathrm{L} / \mathrm{C}$} & $V_{\text {nom, } \boldsymbol{d}}: P<0$ & $a_{1}(P)=\boldsymbol{u}_{\mathbf{1}}\left(P / \boldsymbol{V}_{\boldsymbol{n o m}, \boldsymbol{d}}\right)+\boldsymbol{v}_{\mathbf{1}} n$ & $\bar{\eta}_{d}$ \\
\hline & $\boldsymbol{V}_{\text {nom }, \boldsymbol{c}}: P \geq 0$ & $a_{2}(P)=\boldsymbol{u}_{\mathbf{2}}\left(P / \boldsymbol{V}_{\boldsymbol{n o m}, \boldsymbol{c}}\right)+\boldsymbol{v}_{\mathbf{2}} n$ & $\bar{\eta}_{c}$ \\
\hline \multirow{2}{*}{$C / L / L$} & $V=\int \boldsymbol{V}_{\text {nom,d }}: P<0$ & $a_{1}(P)=\boldsymbol{u}_{\mathbf{1}}\left(P / \boldsymbol{V}_{\boldsymbol{n o m}, \boldsymbol{d}}\right)+\boldsymbol{v}_{\mathbf{1}} n$ & $\eta_{d}(P)=1-P R_{\text {id }} / n V_{\text {nom, } \boldsymbol{d}}^{2}$ \\
\hline & $V= \begin{cases}V_{\text {nom, }} & : P \geq 0\end{cases}$ & $a_{2}(P)=\boldsymbol{u}_{\mathbf{2}}\left(P / \boldsymbol{V}_{\boldsymbol{n o m}, \boldsymbol{c}}\right)+\boldsymbol{v}_{\mathbf{2}} n$ & $\eta_{C}(P)=1-P R_{i c} / n V_{\text {nom }, \boldsymbol{c}}^{2}$ \\
\hline \multirow{2}{*}{ L/L/Q } & \multirow{2}{*}{$V=x_{00}+\frac{x_{10}}{n} l+\frac{x_{01}}{n} b$} & $a_{1}(I)=\boldsymbol{u}_{\mathbf{1}} /+\boldsymbol{v}_{\mathbf{1}} n$ & $\eta_{d}(I, V)=1-I R_{i d} / n \boldsymbol{V}$ \\
\hline & & $a_{2}(l)=\boldsymbol{u}_{\mathbf{2}} /+\boldsymbol{v}_{\mathbf{2}} n$ & $\eta_{C}(I, V)=1-I R_{i c} / n \boldsymbol{V}$ \\
\hline
\end{tabular}

Note: $x_{00}, x_{01}, x_{10}, u_{1}, u_{2}, v_{1}$, and $v_{2}$ are fitted parameters. Bolded parameters are affected by the OR 
limits, and a constant approximation of the voltage. It is linear (see the discussion in "The Benchmark Model" section) and all the equations can be written in terms of $b(k)$ and $P(k)$ only. It has low energy estimation errors when the OR is narrow (Kazhamiaka et al. 2016), and hence it is important to calibrate it with respect to the OR. Treating the benchmark model as a derivative of the PI model makes the calibration procedure (see "Methodology" section) clear, as it utilizes the available spec data, and thereby improves model accuracy. For example, the charging efficiency calculated for an LTO battery with an OR of $[-5 \mathrm{C}, 5 \mathrm{C}]$ is 0.94 , and is increases to $\approx 0.975$ for an OR of [-2 C, 2 C]; for a Lithium-Ferrous-Phosphate $\left(\mathrm{LiFePO}_{4}\right.$ ) battery (A123 Systems 2009), the efficiency for an OR of $[-2 \mathrm{C}, 2 \mathrm{C}]$ is calculated to be $\approx 0.985$.

\section{C/L/C}

This model uses constant approximations of the charging and discharging voltage as well as charging and discharging efficiencies, and uses a linear approximation to the energy content limits. Similarly to the $\mathrm{C} / \mathrm{C} / \mathrm{C}$ model, all the equations can be written in terms of $b(k)$ and $P(k)$ only. Notably, it has the same complexity as the $\mathrm{C} / \mathrm{C} / \mathrm{C}$ model (linear), while offering a better approximation to the energy limits.

C/L/C:

$$
\begin{aligned}
& b(k)=b(k-1)+\Delta_{E}(k) \\
& \Delta_{E}(k)=\left\{\begin{array}{l}
\bar{\eta}_{c} P(k) T_{u}: P(k) \geq 0 \\
\bar{\eta}_{d} P(k) T_{u}: P(k)<0
\end{array}\right. \\
& n \alpha_{d} V_{\text {nom, }} \leq P(k) \leq n \alpha_{c} V_{\text {nom }, c} \\
& u_{1} \frac{P(k)}{V_{\text {nom }, d}}+v_{1} n \leq b(k) \leq u_{2} \frac{P(k)}{V_{\text {nom }, c}}+v_{2} n
\end{aligned}
$$

$C / L / L$

This model uses constant voltage approximations, the same efficiency formula as the PI model, and linear energy content limits. The constant voltage approximation makes the efficiency function linear. Note that in the formulation, we have excluded an explicit $I(k)$ term since, due to the constant voltage approximations, the current is estimated as either $P(k) / V_{\text {nom, }}$ or $P(k) / V_{\text {nom,d }}$. Hence, all the equations can be written in terms of $b(k)$ and $P(k)$. Altogether, this model is quadratic in $P(k)$.

$\mathrm{C} / \mathrm{L} / \mathrm{L}$ :

$$
\begin{array}{r}
b(k)=b(k-1)+\Delta_{E}(k) \\
\Delta_{E}(k)=\left\{\begin{array}{r}
\eta_{c}(P(k)) P(k) T_{u}: P(k) \geq 0 \\
\eta_{d}(P(k)) P(k) T_{u}: P(k)<0
\end{array}\right. \\
\eta_{c}(P(k))=1-\frac{P(k) R_{i c}}{n V_{n o m, c}^{2}} \\
\eta_{d}(P(k))=1-\frac{P(k) R_{i d}}{n V_{n o m, d}^{2}} \\
n \alpha_{d} V_{n o m, d} \leq P(k) \leq n \alpha_{c} V_{n o m, c} \\
u_{1} \frac{P(k)}{V_{\text {nom,d }}}+v_{1} n \leq b(k) \leq u_{2} \frac{P(k)}{V_{n o m, c}}+v_{2} n
\end{array}
$$


$L / L / Q$

This is the most complex of our explicit models. It combines a linear bivariate voltage function, the same quadratic efficiency formula used in the PI model, and a linear approximation for the energy limits. The formulation resembles that of the PI model with the voltage and energy limits replaced by explicit functions. The model is

L/L/Q:

$$
\begin{array}{r}
b(k)=b(k-1)+\Delta_{E}(k) \\
\Delta_{E}(k)=\left\{\begin{array}{r}
\eta_{c}(P(k)) P(k) T_{u}: P(k) \geq 0 \\
\eta_{d}(P(k)) P(k) T_{u}: P(k)<0
\end{array}\right. \\
\eta_{c}(I(k))=1-\frac{I(k) R_{i c}}{n V(k)} \\
\eta_{d}(I(k))=1-\frac{I(k) R_{i d}}{n V(k)} \\
V(k)=x_{00}+\frac{x_{10}}{n} I(k)+\frac{x_{01}}{n} b(k) \\
I(k)=\frac{P(k)}{V(k)} \\
n \alpha_{d} \leq I(k) \leq n \alpha_{c} \\
u_{1} I(k)+v_{1} n \leq b(k) \leq u_{2} I(k)+v_{2} n
\end{array}
$$

Note that all the equations can be written in terms of $b(k), I(k)$ and $P(k)$ and the model is non-linear in both $I(k)$ and $P(k)$. Hence, this model is much more complex and difficult to use.

\section{Evaluation}

In our evaluation, for each of the analytic models discussed in the "Models" section, we quantify the loss of accuracy in the energy content estimate with respect to the PI model. We take this approach because measuring the energy content of a battery directly is very difficult. Hence, we use the PI model to estimate the energy content of the battery, since prior work has found it to accurately model battery dynamics.

More precisely, to evaluate our models, we use LTO (Leclanché 2014) and LithiumFerrous-Phosphate (LiFePO4) (A123 Systems 2009) battery measurements collected while running charge/discharge cycling experiments on these batteries at different $\mathrm{C}$-rates with 2-3 cycles per C-rate, and use the PI model to calculate the energy content of these batteries over the course of the experiment. We then recreate these experiments using simulations of each one of our tractable models, assuming the same applied power that was used in our experiments. We then compare the energy content in the battery over time as simulated by each of our models to the energy content calculated by simulating the battery using the PI model.

\section{Constant-current cycles}

For this section of the evaluation, we calibrate our models over the full operating range of the batteries: [-10 C, $4 \mathrm{C}$ ] for $\mathrm{LiFePO}_{4}$, and [-5 C, $5 \mathrm{C}$ ] for LTO. Figure 5 shows the mean absolute energy error (MAEE) over a full charge (or discharge, for negative $\mathrm{C}$-rates) as a percentage of the maximum battery capacity for each of our analytic models with respect to the PI model. The order of the models from most accurate to least accurate 


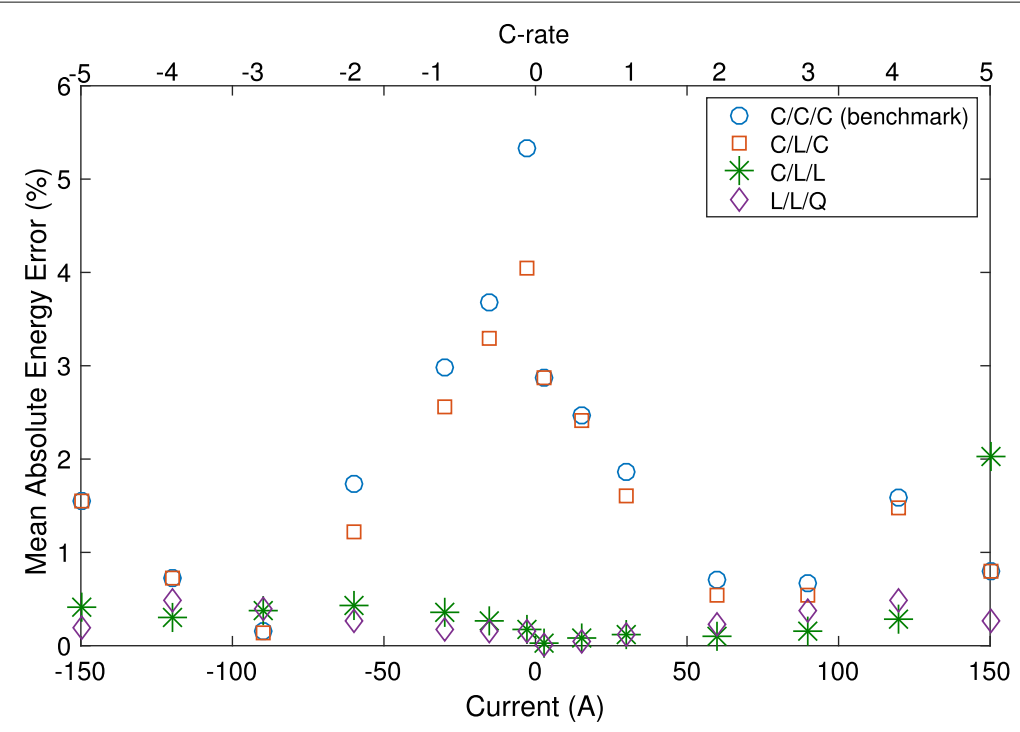

(a) LTO

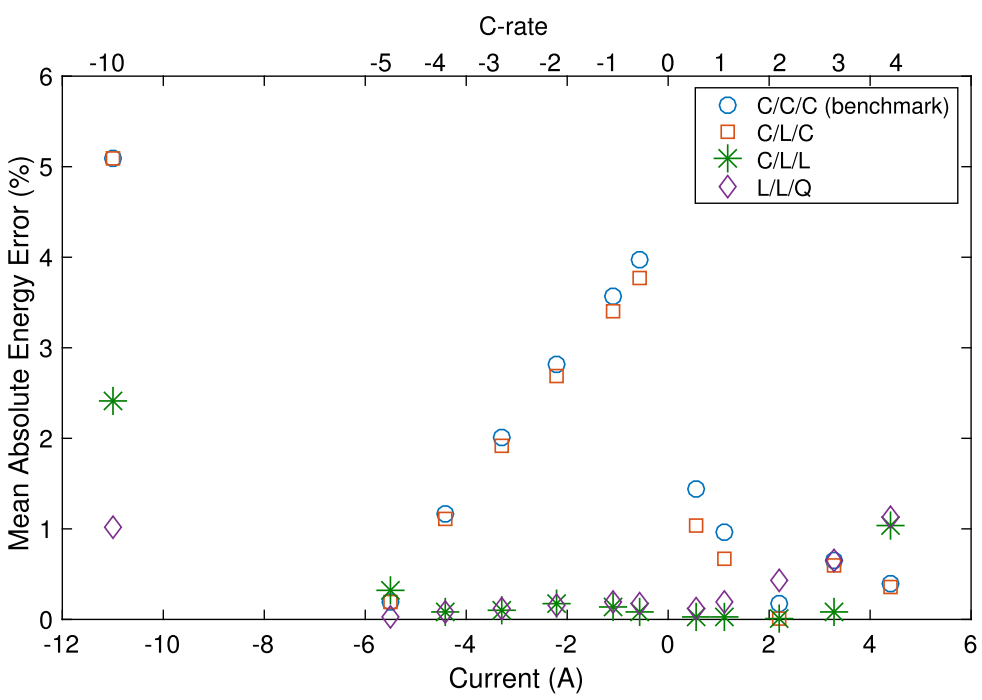

(b) $\mathrm{LiFePO}_{4}$

Fig. 5 Mean absolute energy error for LTO cell (a) and LiFePO4 cell (b) for constant-current cycles at different C-rates (top scale)/Amperes (bottom scale)

mimics their order in terms of complexity. The relative differences in accuracy between the models can be used to understand how much error each approximation contributes. For example, having a linear voltage function $(\mathrm{L} / \mathrm{L} / \mathrm{Q})$ offers little improvement in accuracy over using two constants $(\mathrm{C} / \mathrm{L} / \mathrm{L})$ for all but the most extreme $\mathrm{C}$-rates. $\mathrm{C} / \mathrm{C} / \mathrm{C}$ and $\mathrm{C} / \mathrm{L} / \mathrm{C}$ have low errors around 2-3 $\mathrm{C}$ for LTO for both charging and discharging, and at $5 \mathrm{C}$ discharging and $2 \mathrm{C}$ charging for $\mathrm{LiFePO} 4$ because the constant efficiency approximation happens to be close to the PI model's efficiency at these currents, as shown for an LTO cell in Fig. 4.

It is important to note that errors introduced by two approximations can cancel eachother out. For example, one approximation pushes the model to over-estimate the energy content while another approximation pushes in the other direction. In our results this 
appears to be the case in the $5 \mathrm{C}$ charging test for the LTO battery, where the errors of the $\mathrm{C} / \mathrm{C} / \mathrm{C}$ and $\mathrm{C} / \mathrm{L} / \mathrm{C}$ models are lower than the $\mathrm{C} / \mathrm{L} / \mathrm{L}$ model error.

\section{Varying charge/discharge profile}

To evaluate the accuracy of the models in a realistic setting, we conducted an experiment which mimics the charge/discharge pattern if an LTO battery were installed in a building with photovoltaic (PV) panels, and used as a buffer to store excess PV solar generation and provide power to the building when PV was not enough. We then simulated this system using our analytic models which were calibrated with an OR of $[-2 \mathrm{C}, 2 \mathrm{C}]$, and with the PI model to use as our benchmark. Figure 6 shows the power profile used in our experiment, the resulting energy content evolution over an 8-hour period, as well as the residual with respect to the PI model's energy content estimate. This experiment highlights the effect of the efficiency and voltage function approximations, since the battery did not approach any energy content limits over the course of the test. The residual for all the models stays below $1 \%$ of the total energy capacity of the LTO battery, with the residual of $\mathrm{C} / \mathrm{L} / \mathrm{L}$ and $\mathrm{L} / \mathrm{L} / \mathrm{Q}$ models well below $0.1 \%$.

From these experiments alone, it is unclear if $1 \%$ or $0.1 \%$ residuals are low enough for us to get useful results when these models are used for energy system analysis. Likewise, the MAEE is not easy to relate to the impact of these errors on the actual application of these models. For this reason, we next compare these models in terms of their performance in two case studies.
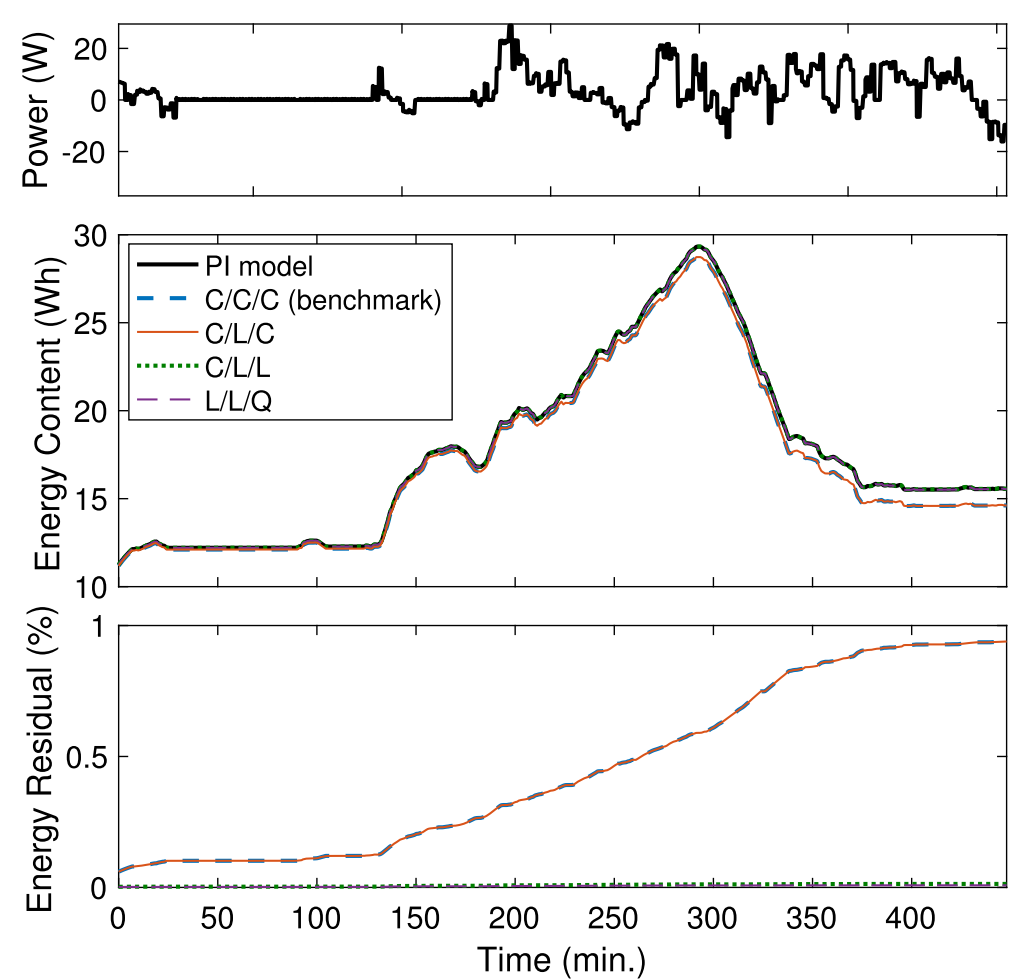

Fig. 6 The energy content over 8 hours in a realistic deployment charge/discharge profile. The residual between the analytic models and the PI model (bottom), as well as the applied power trace (top), are also shown 


\section{Applications}

We performed case studies that compare the accuracy of our models with respect to the PI model for relevant metrics in two battery applications: a solar farm firming application, and grid regulation services. The purpose of these case studies is to exhibit the effects of the choice of battery model on the conclusions that are made. Both case studies are performed via simulations and will be described next.

To obtain results with the proper operating range (OR), which differs for a given application and battery size ${ }^{6}$. We run each simulation twice. First, we calibrate the model with the maximum OR of the battery, i.e., $\left[\alpha_{d}, \alpha_{c}\right]$, and simulate the application. We then observe the OR that was actually used in the simulation, re-calibrate the model, and run the simulation a second time. We report the results from the second simulation. ${ }^{7}$

\section{Solar farm firming}

We consider a small $(100 \mathrm{~kW})$ solar farm participating in an electricity market with hourly power commitments and penalties for failing to adhere to the commitment. Figure 7 shows the basic structure of the system, where $S(t)$ is the incoming solar power which we model using measured data. The load $L(t)$ represents the hourly power commitment that is made by the operator. We assume that the operator always commits to providing the average hourly solar production, and a battery is used to firm the intra-hourly fluctuations in PV production. ${ }^{8}$

An important metric in this application is the amount of energy that was needed from the battery but not delivered, i.e., when not enough PV is being generated to meet the power commitment and the operator needs to discharge the battery but is unable to because the battery does not have enough energy or the required power is too high for the battery to provide. We call this the unmet load on the battery. Choosing a battery size to meet an unmet load target is a classic optimization problem. To see the difference that the choice of battery model will make on the conclusions of this study, we calculate the unmet load by simulating the system with a solar measurement trace for 100 days across a range of battery sizes.

We present results with the model calibrated for the LTO battery chemistry, and remark that we obtained similar results for the $\mathrm{LiFePO}_{4}$ chemistry. Figure 8 shows the unmet load calculated for different battery sizes using the PI model as well as the four analytic models, and the OR used to calibrate model parameters for each battery size. The relative error is very low for the $\mathrm{L} / \mathrm{L} / \mathrm{Q}$ and $\mathrm{C} / \mathrm{L} / \mathrm{L}$ models, and very high for $\mathrm{C} / \mathrm{C} / \mathrm{C}$ and $\mathrm{C} / \mathrm{L} / \mathrm{C}$ models.

The $\mathrm{C} / \mathrm{L} / \mathrm{C}$ model has a low error for 10 and 50 cell batteries, and high error for larger sizes; this is due to the OR and its effect on the different parameters. For smaller battery sizes, the OR is maxed out at $[-5 \mathrm{C}, 5 \mathrm{C}]$, although the actual currents are much more

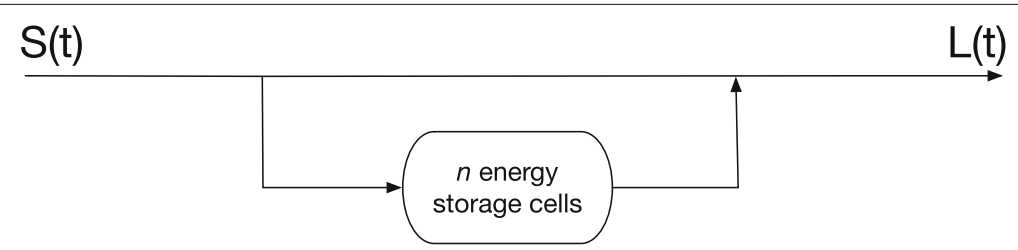

Fig. 7 Power flow diagram in a solar farm equipped with a battery. $S(t)$ is the power generated by the solar cells, and $L(t)$ is the load on the system (power commitment) 

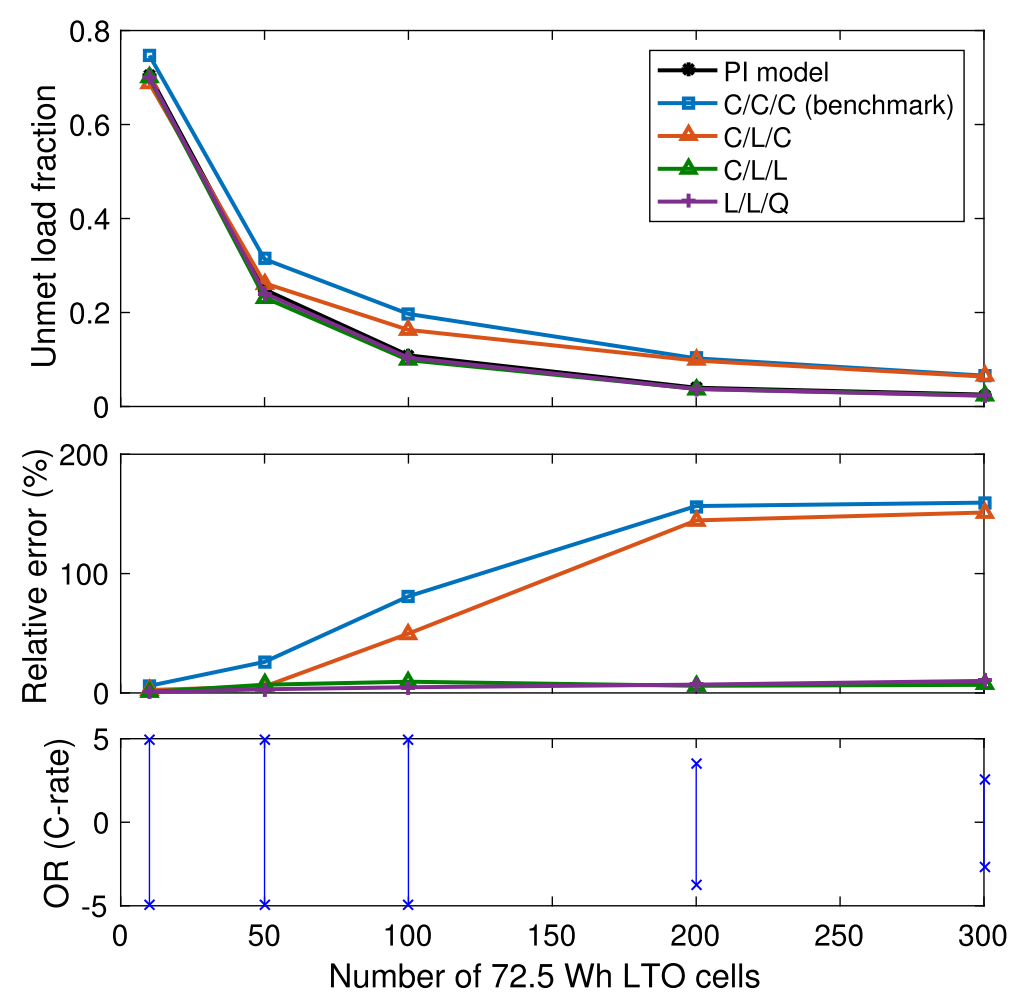

Fig. 8 Unmet load for different battery sizes, computed using five different models. Relative error is with respect to the PI Model, and the effective OR calculated by the PI model is shown in the bottom-most figure

uniformly distributed across the OR with a small (10 - 50 cell) battery compared to the 100-cell battery where the $5 \mathrm{C}$ is a rare occurrence. The OR gradually narrows as the number of cells increases to 300 . For a large OR, it is crucial to model the energy limits as a function of the current, which is why the $\mathrm{C} / \mathrm{L} / \mathrm{C}$ model outperforms the $\mathrm{C} / \mathrm{C} / \mathrm{C}$ model. For narrower OR, the efficiency estimate becomes a more important factor as demonstrated by the high accuracy of $\mathrm{L} / \mathrm{L} / \mathrm{Q}$ and $\mathrm{C} / \mathrm{L} / \mathrm{L}$ models and the similar (poor) accuracy of $\mathrm{C} / \mathrm{L} / \mathrm{C}$ and $\mathrm{C} / \mathrm{C} / \mathrm{C}$ models.

The conclusions of this study can vary greatly depending on the choice of battery model. For example, if the purpose of the study is to determine the smallest battery size that is sufficient to achieve an unmet load target of $40 \%$, all models suggest a similar size. However, for a more realistic $10 \%$ target, the size suggested by the $\mathrm{C} / \mathrm{C} / \mathrm{C}$ and $\mathrm{C} / \mathrm{L} / \mathrm{C}$ models is over $2 x$ larger than the size suggested by the $\mathrm{C} / \mathrm{L} / \mathrm{L}$ and $\mathrm{L} / \mathrm{L} / \mathrm{Q}$ models. The main message is that neither of the linear models works well for this application, and a more complex model such as $\mathrm{C} / \mathrm{L} / \mathrm{L}$ is needed to get accurate results.

\section{Regulation}

We study a grid regulation application ${ }^{9}$ where the battery operator declares the amount of power that they can provide or absorb (by discharging or charging the battery) for the duration of a short-term contract. Given the amount of energy in the battery, the operator needs to calculate the maximum amount of power regulation they could provide in the worst case where their power commitment is requested for the entire duration of the 
contract. In this case study, we model an LTO battery and fix the energy content at the start of the contract to $50 \%$ of the battery's maximum capacity.

We presents the results for down-regulation, where the battery is discharged. Figure 9 shows the power that the battery can consistently provide for various contract durations. We also compute the relative error with respect to the results obtained using the PI model. The $\mathrm{C} / \mathrm{C} / \mathrm{C}$ model has very poor accuracy for all contract lengths, which can be explained by noting that the energy limit is the most important parameter for this application. The $\mathrm{L} / \mathrm{L} / \mathrm{Q}$ model has slightly larger errors (up to $5 \%$ ) than the $\mathrm{C} / \mathrm{L} / \mathrm{C}$ and $\mathrm{C} / \mathrm{L} / \mathrm{L}$ models, which is not an intuitive result and is an important observation; since we are dealing with approximations of multiple components of the PI model, there is room for errors introduced by these components to cancel each other out. Indeed, the improved voltage estimate of the L/L/Q model in turn leads to an accurate current estimate, which is then used to calculate efficiency and energy limits, while the constant voltage approximation in $\mathrm{C} / \mathrm{L} / \mathrm{L}$ and $\mathrm{C} / \mathrm{L} / \mathrm{C}$ models leads to a poor current estimate which actually ends up partially negating the errors in the energy limit approximation. The main message here is that a linear model $(\mathrm{C} / \mathrm{L} / \mathrm{C})$ is accurate enough for this application.

To highlight the effect of the OR on the accuracy of the models, we have calculated the results for the regulation application with a full OR $([-5 \mathrm{C}, 5 \mathrm{C}])$ for all contract lengths. Figure 10 shows the power commitment suggested by the $\mathrm{C} / \mathrm{C} / \mathrm{C}, \mathrm{C} / \mathrm{L} / \mathrm{C}$, and $\mathrm{C} / \mathrm{L} / \mathrm{L}$ models with a full OR, compared to the same models calibrated with the actual OR of the application for each contract length. Every model has worse accuracy when calibrated for the full OR. Note that the L/L/Q model is not shown because its accuracy is almost

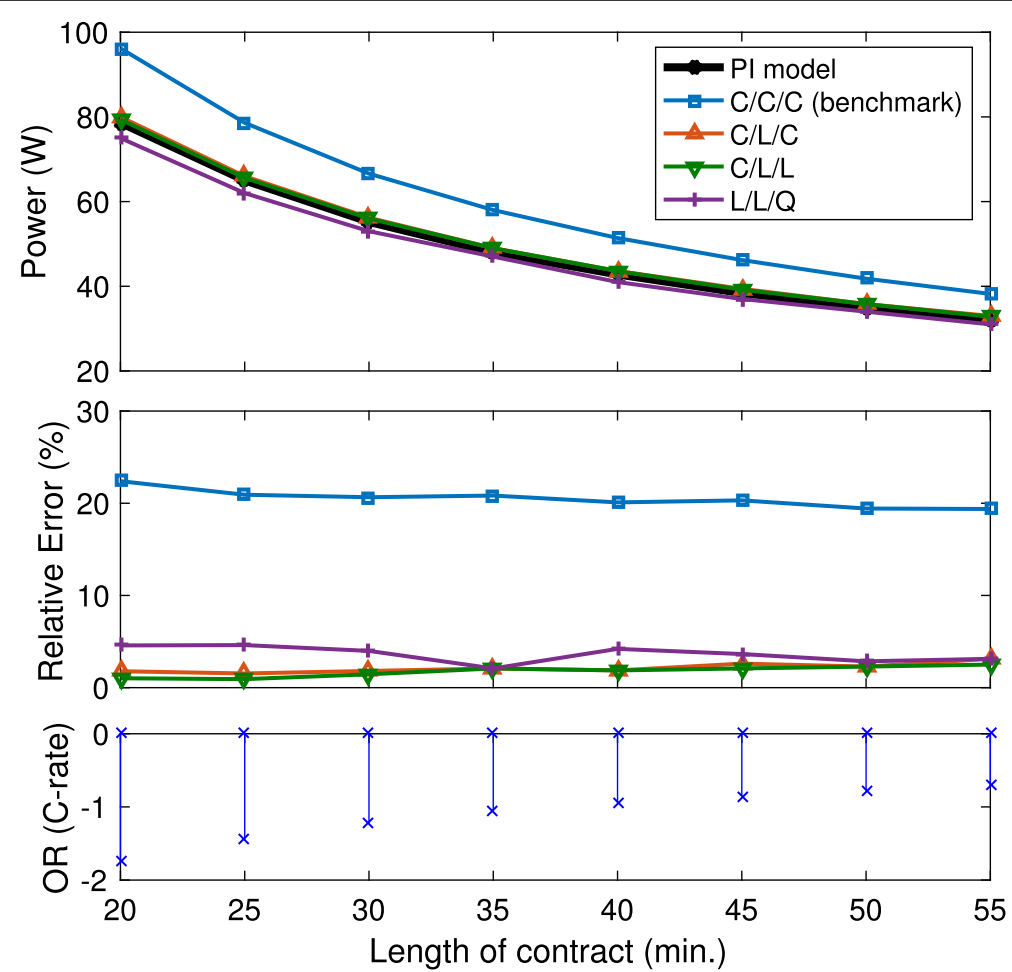

Fig. 9 Maximum power commitment for guaranteed delivery, shown for various contract lengths. The relative error with respect to the PI model is also shown. The OR figure shows the actual OR determined from the PI model simulation 


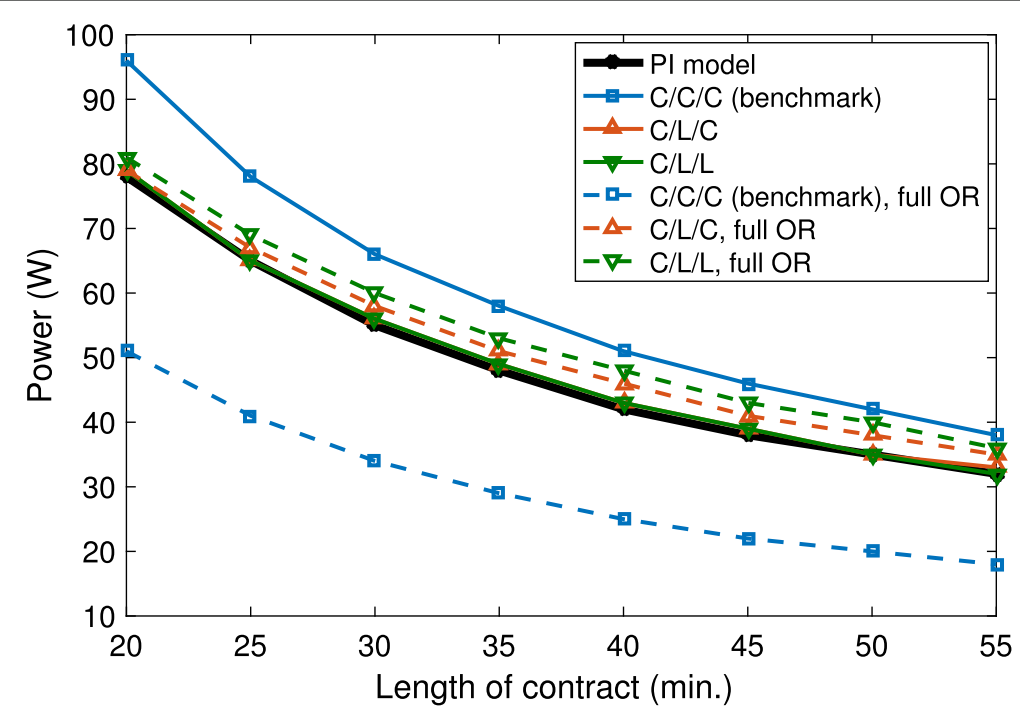

Fig. 10 Computed vs. full OR calibration for the regulation application

unchanged when calibrating for a narrower OR; this is an intuitive result, as the L/L/Q model uses better approximations which are not as sensitive to the OR.

\section{Discussion and Limitations}

Our extensive experimentation with different models and calibration methods has given us insight into how to choose and calibrate a battery model for energy system analysis, in particular for system control and sizing optimization studies. Below, we outline four considerations in selecting a model.

1. What is the size of the optimization problem? For small problems that could be solved by non-linear optimization methods in a reasonable time frame, the L/L/Q and C/L/Q models could be used. Large problems require efficient methods such as linear programming, which require a linear model. In this case, we suggest using the $\mathrm{C} / \mathrm{L} / \mathrm{C}$ model, since it is more accurate than the commonly used $\mathrm{C} / \mathrm{C} / \mathrm{C}$ model, with no added complexity.

2. What battery chemistry is being modelled? Some chemistries, such as $\mathrm{LiFePO}_{4}$, have "flat" voltage profiles which can be approximated well as a constant voltage. Cells with very low internal impedance have high efficiency values (close to 1) which could be closely approximated using a constant. The area under the voltage curve for different $\mathrm{C}$-rates on a voltage vs. charge graph (commonly found in a spec sheet) can hint at the shape of the energy limit function ${ }^{10}$. This information can help guide the choice of battery model.

3. Which constraints of the battery is the application most sensitive to? This may or may not be known a priori; if it is known, it can guide the selection of the model based on the effectiveness of the approximations to each model component. For example, if efficiency is a crucial parameter, we suggest the use of the $\mathrm{L} / \mathrm{L} / \mathrm{Q}$ or $\mathrm{C} / \mathrm{L} / \mathrm{L}$ models which have more accurate efficiency functions than the $\mathrm{C} / \mathrm{L} / \mathrm{C}$ model. 
4. What is the operating range of the application? If it is known a priori, it should be used to calculate the parameters of the battery. If it is unknown, the analysis should first be conducted with the model calibrated using the maximum OR that the battery allows, keeping track of the OR that is actually used by the battery in the analysis. Then, the analysis should be re-run with parameters computed for the OR observed in the first analysis. This can be repeated until the calibrated OR matches the observed OR; in our experience, we noticed that there was little benefit in going beyond one or two iterations of this method.

Our work has two main limitations. The first is that we have only evaluated our model on two Lithium-ion battery chemistries; we defer the testing of a broader range of electrochemical storage technologies to future work. Finally, there are limitations to the PI model that are inherited by the tractable models we have derived: it does not model the effects of temperature on the battery, and does not take the battery state of health and degradation into account.

\section{Conclusion}

We propose three novel, analytical, and easily-calibrated battery models which are derived from the PI model: the $\mathrm{C} / \mathrm{L} / \mathrm{C}, \mathrm{C} / \mathrm{L} / \mathrm{L}$, and $\mathrm{L} / \mathrm{L} / \mathrm{Q}$ models. These models were obtained by using a methodology that involves measuring the effect of different degrees of approximations to complex and non-analytic PI model components, and selecting the models with a desirable trade-off between accuracy and complexity. In our evaluation, we compare the accuracy of the derived models in three settings: constant-current cycling, a real-world charge/discharge profile, and two energy system case studies. In particular, we show that the $\mathrm{C} / \mathrm{L} / \mathrm{C}$ model is more accurate and just as tractable as the benchmark model for optimization studies. We also show that the choice of model can significantly affect the conclusions of model-based energy system studies and that sometimes a non linear model $(\mathrm{C} / \mathrm{L} / \mathrm{L})$ is necessary. Finally, we show that the operating range of the battery is a crucial parameter for obtaining accurate results with simplified models.

\section{Endnotes}

${ }^{1}$ PI stands for "Power-based Integrated", because it uses power as input and integrates the BMS and cell models into a single model.

${ }^{2}$ If the spec sheet provides only one value for internal impedance, then we use that value for both $R_{i c}$ and $R_{i d}$.

${ }^{3}$ To scale these equations to more than one cell, we divide $I(k)$ and $b(k)$ by $n$ wherever they appear.

${ }^{4}$ We use MATLAB's fit function to compute the linear approximation.

${ }^{5}$ Technically, the voltage approximation given in Eq. 13 corresponds to two constants instead of one.

${ }^{6}$ The effect of the battery size on the OR, which is given in terms of C-rate, can be explained concisely via example: an application may require a current of $100 \mathrm{Amps}$, which equates to $1 \mathrm{C}$ for a particular battery, but only $0.5 \mathrm{C}$ when the battery's size is doubled

${ }^{7}$ One could continue re-running simulations using the OR observed in the previous simulation until the OR used to obtain parameters and the OR observed in the simulation converge. In practice, we found that one iteration of this method is enough to get a reasonable OR. 
${ }^{8}$ A comprehensive study of this system can be found in Ghiassi-Farrokhfal et al. (2015).

${ }^{9} \mathrm{~A}$ comprehensive study of this application can be found in Fooladivanda et al. (2016)

${ }^{10}$ The area under a discharging voltage vs. charge curve is equal to the energy discharged from the battery at the given $\mathrm{C}$-rate.

\section{Abbreviations}

BMS: Battery management system; LP: Linear program; LTO: Lithium-titanate; LiFePO4: Lithium-ferrous-phosphate; MAEE: Mean absolute energy error; OR: Operating range; PI: Power-based integrated battery model; PV: Photovoltaic

\section{Acknowledgements}

We acknowledge Prof. Karl-Heinz Pettinger and the technicians at the Ruhstorf Technology Center for Energy for helping us run the experiments that contributed to the evaluation of our models.

\section{Funding}

None to declare.

\section{Availability of data and materials}

The data sets used to drive the evaluation of the models are available from the corresponding author on reasonable request.

\section{Authors' contributions}

All of the authors worked together to conceive the ideas summarized in this paper, and to write and review the paper. The implementation of the models in MATLAB, their evaluation, and application testing was done by FK. All authors read and approved the final manuscript.

\section{Competing interests}

The authors declare that they have no competing interests.

\section{Publisher's Note}

Springer Nature remains neutral with regard to jurisdictional claims in published maps and institutional affiliations.

Received: 6 February 2019 Accepted: 12 April 2019

Published online: 07 May 2019

\section{References}

A123 Systems (2009) High Power Lithium Ion APR18650M1A. A123 Systems. LiFePO4 cell specifications. https://blizzard. cs.uwaterloo.ca/iss4e/wp-content/uploads/2019/04/A123-APR18650M1A.pdf

Atia R, Yamada N (2016) Sizing and analysis of renewable energy and battery systems in residential microgrids. IEEE Trans Smart Grid 7(3):1204-1213

Chen M, Rincon-Mora GA (2006) Accurate electrical battery model capable of predicting runtime and iv performance. IEEE Trans Energy Convers 21(2):504-511

Chen S, Gooi HB, Wang M (2012) Sizing of energy storage for microgrids. IEEE Trans Smart Grid 3(1):142-151

Einhorn M, Conte FV, Kral C, Fleig J (2013) Comparison, selection, and parameterization of electrical battery models for automotive applications. IEEE Trans Power Electron 28(3):1429-1437

Fooladivanda D, Rosenberg C, Garg S (2016) Energy storage and regulation: an analysis. IEEE Trans Smart Grid 7(4):1813-1823

Gearhart JL, Adair KL, Detry RJ, Durfee JD, Jones KA, Martin N (2013) Comparison of open-source linear programming solvers. Tech. Rep. SAND2013-8847

Ghiassi-Farrokhfal Y, Kazhamiaka F, Rosenberg C, Keshav S (2015) Optimal design of solar pv farms with storage. IEEE Trans Sustain Energy 6(4):1586-1593

Ghiassi-Farrokhfal Y, Rosenberg C, Keshav S, Adjaho M-B (2016) Joint optimal design and operation of hybrid energy storage systems. IEEE Journal Sel Areas Commun 34(3):639-650

Hassan AS, Cipcigan L, Jenkins N (2017) Optimal battery storage operation for pv systems with tariff incentives. Appl Energy 203:422-441

He H, Xiong R, Zhang X, Sun F, Fan J (2011) State-of-charge estimation of the lithium-ion battery using an adaptive extended kalman filter based on an improved thevenin model. IEEE Trans Veh Technol 60(4):1461-1469

Hentunen A, Lehmuspelto T, Suomela J (2014) Time-domain parameter extraction method for thévenin-equivalent circuit battery models. IEEE Trans Energy Convers 29(3):558-566

Hu X, Li S, Peng H (2012) A comparative study of equivalent circuit models for li-ion batteries. J Power Sources 198:359-367

Jackey R, Saginaw M, Sanghvi P, Gazzarri J, Huria T, Ceraolo M (2013) Battery model parameter estimation using a layered technique: an example using a lithium iron phosphate cell. Technical report. SAE Technical Paper

Kazhamiaka F, Keshav S, Rosenberg C, Pettinger K-H (2018) Simple spec-based modelling of lithium-ion batteries. IEEE Trans Energy Convers 33:1757-1765

Kazhamiaka F, Rosenberg C, Keshav S, Pettinger K-H (2016) Li-ion storage models for energy system optimization: the accuracy-tractability tradeoff. In: Proceedings of the Seventh International Conference on Future Energy Systems. ACM. p 17

Kendall DG (1953) Stochastic processes occurring in the theory of queues and their analysis by the method of the imbedded markov chain. Ann Math Stat:338-354

Khalilpour R, Vassallo A (2016) Planning and operation scheduling of pv-battery systems: A novel methodology. Renew Sust Energ Rev 53:194-208 
Klein R, Chaturvedi NA, Christensen J, Ahmed J, Findeisen R, Kojic A (2013) Electrochemical model based observer design for a lithium-ion battery. IEEE Trans Control Syst Technol 21(2):289-301

Leclanché (2014) LecCell 30Ah High Energy. Leclanché. Lithium-Titanate cell specifications. https://blizzard.cs.uwaterloo. ca/iss4e/wp-content/uploads/2019/04/leccell-30ah-high-energy.pdf

Mehrabankhomartash M, Rayati M, Sheikhi A, Ranjbar AM (2017) Practical battery size optimization of a pv system by considering individual customer damage function. Renew Sust Energ Rev 67:36-50

Scrosati B, Hassoun J, Sun Y-K (2011) Lithium-ion batteries. a look into the future. Energy Environ Sci 4(9):3287-3295

Tanim TR, Rahn CD, Wang C-Y (2015) A temperature dependent, single particle, lithium ion cell model including electrolyte diffusion. J Dyn Syst Meas Control 137(1):011005

Zou C, Manzie C, Nešić D (2016) A framework for simplification of pde-based lithium-ion battery models. IEEE Trans Control Syst Technol 24(5):1594-1609

Submit your manuscript to a SpringerOpen ${ }^{\mathcal{D}}$ journal and benefit from:

- Convenient online submission

- Rigorous peer review

- Immediate publication on acceptance

- Open access: articles freely available online

- High visibility within the field

Retaining the copyright to your article

Submit your next manuscript at $\boldsymbol{\triangleright}$ springeropen.com 